\title{
La trisomía 21 y la enfermedad por coronavirus de 2019 (COVID-19)
}

\author{
Trisomy 21 and the coronavirus disease 2019 (COVID-19)
}

La trisomía 21 es una entidad clínica, debida a la alteración cromosómica más común presente en los nacidos vivos. ${ }^{1}$ Transcurre con discapacidad intelectual, afecciones médicas, como el hipotiroidismo, una amplia variedad de dismorfias craneofaciales y malformaciones congénitas. ${ }^{2}$ La esperanza de vida en los individuos con esta entidad genética ha aumentado significativamente en las últimas décadas, aunque esta población tiene un mayor riesgo de mortalidad neonatal e infantil en relación con sus pares sin la alteración cromosómica (el 1,65 \% vs. el 0,36 \% y el $4 \%$ vs. el $0,48 \%$ ), respectivamente. ${ }^{1}$ Las principales causas de mortalidad son las cardiopatías congénitas, ${ }^{1,2}$ las alteraciones respiratorias, en el sistema nervioso, en el tracto gastrointestinal, en el genitourinario, en el aparato musculoesquelético, ${ }^{1}$ leucemia, ${ }^{1,2}$ cáncer testicular y sepsis. ${ }^{1}$

Los individuos con esta entidad genética pueden presentar síntomas respiratorios crónicos y graves con más frecuencia, porque se asocian, como se hizo referencia, a anomalías en las vías respiratorias,,$^{1,3}$ cardiopatías congénitas, hipotonía generalizada y disfunción en la deglución. ${ }^{1}$ Además, estas alteraciones en las vías respiratorias pueden ser combinadas en la mayoría de los casos y todo esto predispone a infecciones respiratorias recurrentes, que representan la segunda causa de mortalidad indistintamente de la edad. ${ }^{1,3,4}$

Además, los individuos con trisomía 21 padecen, con más frecuencia, defectos inmunológicos, ${ }^{5}$ entre los cuales se pueden citar alteraciones en la inmunidad innata y adaptativa, anomalías en linfocitos $\mathrm{T}$ y $\mathrm{B}, 1,5$ defecto primario en el compartimento de las células $B$, caracterizado por un reducido número en sangre periférica, especialmente, de las células de memoria y de la inmunoglobulina $M$, que representan la primera línea de defensa contra las infecciones. ${ }^{1}$

En cuanto a la ya comentada inmunidad innata, alteraciones en la función de las células T gamma delta, el inflamasoma y los receptores similares a Toll, como sus vías, pueden intervenir en una adecuada repuesta.
Adicionalmente, alteraciones en los monocitos, quimiotaxis en los neutrófilos, citoquinas circulantes y anormalidad en las respuestas de los anticuerpos contribuyen a un mayor riesgo de infecciones y, a su vez, están relacionados con mal pronóstico. ${ }^{5}$ También se ha demostrado que responden mal a la inmunización primaria y su protección puede requerir protocolos de vacunación personalizados. Actualmente, la Academia Americana de Pediatría recomienda la vacuna contra el neumococo, la influenza y el uso de palivizumab, ya que corren el riesgo de contraer infecciones graves por el virus sincicial respiratorio. ${ }^{1}$

El reciente brote de una neumonía grave asociada con un nuevo coronavirus (SARS-CoV-2, por las siglas en inglés de síndrome respiratorio agudo grave por coronavirus 2, o COVID-19, por enfermedad por coronavirus de 2019,) creó una emergencia a nivel mundial, que ha puesto a las instituciones de salud pública del mundo en alerta máxima. ${ }^{6}$ En la actualidad, se plantea un desafío global, en el que la información clínica y terapéutica sigue siendo limitada, lo que amerita una constate actualización científica.

Por lo tanto, las medidas preventivas mantienen un rol importante para controlar y evitar la propagación de esta enfermedad y más en individuos con alteraciones en las vías respiratorias y otros factores de riesgo ya mencionados. ${ }^{6}$ Aunque estas medidas son ampliamente difundidas por los medios de comunicación, redes sociales y otras fuentes, paradójicamente, se cometen errores que se pueden evitar con facilidad. Estas medidas deben ser seguidas no solo por el individuo con trisomía 21, sino por los familiares y todas aquellas personas que mantienen contacto con este, lo que, tal vez, sea considerado uno de los principales errores. Se debe evitar, además, una exposición innecesaria y, con ello, se hace énfasis en la principal recomendación de quedarse en casa. Desde el hogar, debe surgir la creatividad para aprovechar el tiempo y hacerlo más llevadero. Esta batalla es de todos, y unidos es la única forma de volver lo más pronto posible a la tan anhelada normalidad. 
Las diferentes fundaciones para la trisomía 21 han participado de forma proactiva en la promulgación de la información y esto sirve de apoyo a las familias. Por lo tanto, el mensaje no debe ser de alarma, sino previsor. La participación de personas con la entidad en diferentes campañas tiene como objetivo crear mayor conciencia y sensibilidad en la población general, que es aquella que tiene, a su vez, el deber de velar y cuidar el bienestar de los individuos con esta enfermedad genética.

En el mundo, existen más de mil millones de personas que viven con algún tipo de discapacidad y es probable que la pandemia ocasionada por el SARS-CoV-2 (COVID-19) afecte mayormente a estos individuos, lo que exhibe un mayor riesgo de morbilidad y mortalidad. Todo esto resalta la necesidad urgente de mejorar la prestación de servicios de salud para este grupo de pacientes, hacerlo más accesible y convertirlo en prioridad. En particular, en los países de bajos y medianos ingresos, la capacidad de dar respuesta puede verse afectada ante la falta de planificación, preparación y poder brindar una respuesta verdaderamente inclusiva y accesible a esta población vulnerable. ${ }^{7}$

Según la Revista Virtual Sindrome de Down, ${ }^{8}$ se sabe que algunos individuos ya han presentado la infección con el SARS-CoV-2 (COVID-19) con evolución variable, desde la recuperación hasta el fallecimiento. Con esto, surge la interrogante de si la población con trisomía 21 se ve afectada con más frecuencia que el resto de la población y si se presenta de forma más grave, por lo que su estudio apenas se inicia. ${ }^{8}$

Ante las características descritas en la trisomía 21, esta población debe ser considerada de riesgo indistintamente de su edad, no solo para SARSCoV-2 (COVID-19), sino para otros agentes infecciosos ya descritos. Su manejo debe ser específico e individualizado, pues se necesita una evaluación y un seguimiento apropiado.

Se debe, por lo tanto, tener en cuenta un adecuado empleo de los fármacos, inmunizaciones y agentes profilácticos que pueden ser útiles con el objeto de disminuir las diferentes complicaciones que pueden exhibir estos individuos. Por ende, los pediatras, así como todo el personal de salud, de forma general, deben comprometerse y esforzarse por recopilar los datos clínicos necesarios, que incluyen el muestreo de los casos, con el objeto de ampliar la comprensión de las características clínicas, la prevención, la patogénesis del agente infeccioso y la respuesta del huésped. ${ }^{9}$

\section{Dedicación:}

Este artículo está dedicado a las personas con trisomía 21, a las que nos debemos para su bienestar y cuidado.

A la "fuerza blanca", todo aquel equipo de salud que, con el conocimiento y valentía, luchan en esta compleja batalla.

\section{Prof. Francisco Cammarata-Scalisi ${ }^{a}$, Prof. Dr. Antonio Cárdenas Tadich, Dr. Marco Medina $a^{a}$ y Dr. Michele Callea ${ }^{b}$}

a. Servicio de Pediatría, Hospital Regional de Antofagasta, Chile.

b. Unit of Dentistry, Bambino Gesù Children's Hospital, IRCCS, Rome, Italy.

francocammarata19@gmail.com, dr_cardenas2000@yahoo.es.

http:/ / dx.doi.org/10.5546/ aap.2020.230

Texto completo en inglés:

http:/ / dx.doi.org/10.5546/ aap.2020.eng.230

Cómo citar: Cammarata-Scalisi F, Cárdenas Tadich A, Medina M, Callea M. La trisomía 21 y la enfermedad por coronavirus de 2019 (COVID-19). Arch Argent Pediatr 2020;118(4):230-231.

\section{REFERENCIAS}

1. ValentiniD, DiCamilloC, MiranteN, Marcelini V, etal.Effects of Pidotimod on recurrent respiratory infections in children with Down syndrome: a retrospective Italian study. Ital J Pediatr. 2020; 46(1):31.

2. Fisher PG. Who should care for children with Down syndrome? J Pediatr. 2020; 218:1-4.

3. De Lausnay M, Verhulst S, Boel L, Wojciechowski M, et al. The prevalence of lower airway anomalies in children with Down syndrome compared to controls. Pediatr Pulmonol. 2020 [Epub ahead of print].

4. Kusumoto Y, Imai K, Ohyama Y, Fukayama H, et al. Oral management of a patient with Down syndrome and agammaglobulinemia: a case report. BMC Oral Health. 2020; 20(1):71.

5. Huggard D, Doherty DG, Molloy EJ. Immune dysregulation in children with Down syndrome. Front Pediatr. 2020; 8:73.

6. Marraro GA, Spada C. Consideration of the respiratory support strategy of severe acute respiratory failure caused by SARS-CoV-2 infection in children. Zhongguo Dang Dai Er Ke Za Zhi. 2020; 22(3):183-94.

7. Armitage R, Nellums LB. The COVID-19 response must be disability inclusive. Lancet Public Health. 2020 [Epub ahead of print].

8. COVID-19 Nadie lo había invitado. Revista Virtual Síndrome de Down. 2020; 227. [Acceso: 3 de abril de 2020]. Disponible en: https: / / www.down21.org/revista-virtual/1774-revistavirtual-sindrome-de-down-2020 / revista-virtual-abril-2020n-227/3436-covid-19-nadie-lo-habia-invitado.html.

9. Britton PN, Marais BJ. Questions raised by COVID-19 case descriptions. J Paediatr Child Health. 2020 [Epub ahead of print]. 